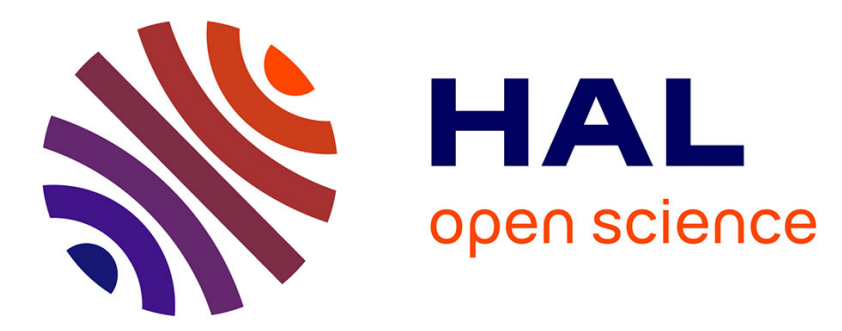

\title{
The cryo-EM structure of the chloroplast ClpP complex
}

Ning Wang, Yifan Wang, Qian Zhao, Xiang Zhang, Chao Peng, Wenjuan Zhang, Yanan Liu, Olivier Vallon, Michael Schroda, Yao Cong, et al.

\section{To cite this version:}

Ning Wang, Yifan Wang, Qian Zhao, Xiang Zhang, Chao Peng, et al.. The cryo-EM structure of the chloroplast ClpP complex. Nature Plants, 2021, 7 (11), pp.1505 - 1515. 10.1038/s41477-021-01020-x . hal-03452775

\section{HAL Id: hal-03452775 \\ https://hal.science/hal-03452775}

Submitted on 14 Dec 2021

HAL is a multi-disciplinary open access archive for the deposit and dissemination of scientific research documents, whether they are published or not. The documents may come from teaching and research institutions in France or abroad, or from public or private research centers.
L'archive ouverte pluridisciplinaire HAL, est destinée au dépôt et à la diffusion de documents scientifiques de niveau recherche, publiés ou non, émanant des établissements d'enseignement et de recherche français ou étrangers, des laboratoires publics ou privés. 
2 Ning Wang ${ }^{1,2 *}$, Yifan Wang ${ }^{2,3, *}$, Qian Zhao ${ }^{1}$, Xiang Zhang ${ }^{3}$, Chao Peng ${ }^{4}$, Wenjuan

3 Zhang ${ }^{1,2}$, Yanan Liu ${ }^{1,2}$, Olivier Vallon ${ }^{5}$, Michael Schroda ${ }^{6}$, Yao Cong ${ }^{3, \#}$, Cuimin Liu ${ }^{1,2, \#}$

$5{ }^{1}$ State Key Laboratory of Plant Cell and Chromosome Engineering, Institute of 6 Genetics and Developmental Biology. The Innovative Academy of Seed Design,

7 Chinese Academy of Sciences, Beijing, 100101, China

$8 \quad{ }^{2}$ University of Chinese Academy of Sciences, Beijing, 100101, China

$9{ }^{3}$ State Key Laboratory of Molecular Biology, National Center for Protein Science 10 Shanghai, Shanghai Institute of Biochemistry and Cell Biology, Center for Excellence 11 in Molecular Cell Science, Chinese Academy of Sciences, Shanghai 200031, China; 12 Shanghai Science Research Center, Chinese Academy of Sciences, Shanghai, China $13 \quad 201210$

$14{ }^{4}$ National Facility for Protein Science in Shanghai, Zhangjiang Lab, Shanghai 15 Advanced Research Institute, CAS, Shanghai 201210, China

${ }^{5}$ UMR7141 CNRS-Sorbonne Université, Institut de Biologie Physico-Chimique, Paris 75005, France

${ }^{6}$ Molecular Biotechnology \& Systems Biology, TU Kaiserslautern, Paul-Ehrlich Straße 23, D-67663 Kaiserslautern, Germany

$21 *$ these authors contributed equally to this work

\# Correspondence to: cmliu@genetics.ac.cn; $\underline{\text { cong@sibcb.ac.cn }}$ 


\section{Abstract}

Protein homeostasis in plastids is strategically regulated by the protein quality control system involving multiple chaperones and proteases, among them the Clp protease. Here we determined the structure of the chloroplast ClpP complex from Chlamydomonas reinhardtiiby cryo-EM. ClpP contains two heptameric catalytic rings without any symmetry. The top ring contains one ClpR6, three ClpP4 and three ClpP5 subunits while the bottom ring is composed of three $\mathrm{ClpP} 1_{\mathrm{C}}$ subunits and one each of the ClpR1-4 subunits. ClpR3, ClpR4 and ClpT4 subunits connect the two rings and stabilize the complex. The chloroplast Cpn11/20/23 co-chaperonin, a co-factor of Cpn60, forms a cap on the top of ClpP by protruding mobile loops into hydrophobic clefts at the surface of the top ring. The co-chaperonin repressed ClpP proteolytic activity in vitro. By regulating Cpn60 chaperone and ClpP protease activity, the cochaperonin may play a role in coordinating protein folding and degradation in the chloroplast.

\section{INTRODUCTION}

Sophisticated pathways within cells control and regulate the biogenesis, trafficking and degradation of proteins to ensure protein homeostasis (proteostasis). These pathways belong to the protein quality control (PQC) system that involves the participation of various chaperones and proteases $^{1-5}$. Molecular chaperones act as key components of the PQC system. They assist protein folding when new polypeptide chains emerge from ribosomes, when proteins have translocated through membranes, and when proteins become misfolded. Proteases are another important component of the PQC system that recognize and degrade substrate proteins which cannot fold or refold to the native state.

The molecular chaperone chaperonin is a tetradecameric cylinder formed by two rings stacked back to back, whose central cavity is used to shield a bound unfolded protein from the crowded environment and to assist its folding to the native state in an ATP-dependent process ${ }^{6}$. A co-chaperonin complex seals the central cavity of group I 
chaperonins to form the isolated folding environment. In bacteria, this lid is formed by the homo-heptameric GroES co-chaperonins. In plastids, two types of co-chaperonin subunits exist, Cpn10 and Cpn20, the latter formed by two co-chaperonin domains in tandem. In addition to its role as a co-chaperonin for Cpn60, Cpn20 has been shown in Arabidopsis to mediate superoxide dismutase (FeSOD) activation independent of the Cpn60 chaperonin ${ }^{7}$. In addition, the expression level of the Cpn20 gene influenced the physiological function of $\mathrm{ABA}$ signaling during seed germination and promoted stomatal closure without Cpn60 involvement ${ }^{8,9}$. These results indicate that the Cpn20 protein might exhibit biochemical functions in addition to that as a co-factor for Cpn60 in protein folding.

In bacterial cells or endosymbiotic organelles, proteins are degraded by many types of proteases including serine-, aspartate-, and threonine-proteases ${ }^{10,11}$. The Clp protease is a conserved serine protease that consists of the ClpP core complex and assistant chaperones ${ }^{12}$. The first crystal structure of the E. coli ClpP core complex revealed a barrel composed of two stacked heptameric rings, with one catalytic triad in each subunit ${ }^{13}$. The degradation of protein substrates by the Clp protease in E. coli is assisted by two hexameric AAA+ (ATPase Associated with multiple cellular Activities) chaperones, $\mathrm{ClpX}$ and $\mathrm{ClpA}$, both of which can bind to the ClpP core complex via one of its surfaces ${ }^{14}$. Fueled by ATP hydrolysis, substrate proteins are unfolded by ClpX or ClpA and threaded into the central cavity of the ClpP core complex ${ }^{14}$, where the substrate is degraded. Recent studies have explored the functional significance of the symmetry mismatch between the AAA+ chaperone hexamer and the heptamer of the ClpP core complex in E. coli ${ }^{15-18}$. In particular, it has been proposed to induce a rotational movement between the AAA+ ATPase and the ClpP core and to participate in substrate transport ${ }^{16}$.

In mitochondria, the ClpP core consists of 14 identical subunits that are similar to their bacterial homologs ${ }^{19}$. In contrast, the ClpP core complex in chloroplasts is made up of three types of subunits termed ClpP, ClpR, and ClpT. ClpR subunits are homologous to ClpP but cannot contribute to catalysis because they lack one or more 
of the active residues of the Ser-His-Asp catalytic triad. ClpT proteins are completely different proteins of around $20 \mathrm{kDa}$ that share homology with the $\mathrm{N}$-terminal domain of the AAA+ chaperones. ClpT subunits have been proposed to link the two heptameric rings and thus maintain the stability of the tetradecameric $\mathrm{ClpP}$ core ${ }^{20-23}$. In the green alga Chlamydomonas reinhardtii, the subunits of the chloroplast ClpP core complex are encoded by three $C l p P$ genes ( $\operatorname{lpP} 1, C L P P 4, C L P P 5$, the former chloroplast-encoded), five $C l p R$ genes (CLPR1-4, CLPR6) and two ClpT genes (CLPT3, CLPT4) ${ }^{24,25}$. The plastidial $\operatorname{clpP} 1$ gene produces $\mathrm{ClpP} 1_{\mathrm{H}}$, which can be further processed by unknown peptidases to generate $\mathrm{ClpP} 1_{\mathrm{N}}, \mathrm{ClpP} 1_{\mathrm{C}}$ and $\mathrm{ClpP} 1_{\mathrm{C}}$, subunits which are part of the $\mathrm{ClpP}$ core complex ${ }^{26}$. Translational attenuation of clpPl in Chlamydomonas led to the stabilization of misassembled photosynthetic enzymes ${ }^{27}$, while its conditional repression caused serious autophagy responses and activated the protein quality control system ${ }^{28}$. In Arabidopsis chloroplasts, the ClpP complex consists of five ClpP type subunits (ClpP3-6 and ClpP1), four ClpR type subunits (ClpR1-4) and two ClpT type subunits (ClpT1-2) 20, 22, 29. Loss of ClpP5 gene function was embryo-lethal ${ }^{30}$. Although the R-type subunits were considered unable to degrade substrate proteins, either $C l p R 2$ or $C l p R 4$ gene knockout resulted in delayed embryogenesis suggesting their functional importance ${ }^{30}$. These results indicate that the Clp protease plays an essential role in maintaining protein homeostasis in chloroplasts, even though some subunits can be substituted by others. It was observed previously that the co-chaperonin Cpn20 interacts with the ClpP complex ${ }^{20}$, but how this interaction takes place and whether it has functional implications are not known.

Recently, several structures of the Clp machinery composed of core complex and $\mathrm{AAA}+$ chaperone have been resolved by cryo-EM. However these studies focused on bacterial Clp machineries, while there is no high-resolution structure of chloroplast Clp, yet ${ }^{15-17,31}$. In this study, we solved the cryo-EM structure of the Chlamydomonas chloroplast ClpP core complex. The complex is asymmetrical and polypeptide chains could be assigned to a known Clp gene. Furthermore, the co-chaperonin complex was observed to bind to the top ring of the $\mathrm{ClpP}$ core without inducing a major 
conformational change. We hypothesize that co-chaperonins act as regulatory factors in chloroplast proteostasis.

\section{RESULTS}

\section{Co-chaperonins inhibit CIpP proteolytic activity}

In previous work, the ClpP complex has been purified from Chlamydomonas reinhardtii chloroplasts by Strep-tag affinity purification and the complex subunits have been assigned to P-, R- and T-type by mass spectrometry ${ }^{32}$. Based on that work, we improved the purification strategy by combining affinity purification with multiple chromatography steps to obtain large amounts of highly purified Chlamydomonas ClpP complexes. The purified complexes were separated on a 12\%-18\% SDS gel and on a native gel and proteins were visualized by Coomassie staining (Figs. 1A and 1B). In the native gel, the purified ClpP complex migrated as a diffuse band with a molecular mass below the 820 kDa of oligomeric Cpn60 (Fig. 1B). By Asymmetric Flow Field Flow Fractionation (AFFFF) we calculated a molecular mass of $549 \mathrm{kDa}$ for the ClpP complex, which is much larger than the $240 \mathrm{kDa}$ of E. coli ClpP (Extended Data Fig. 1A). Each visible protein band in the SDS gel was cut out and analyzed by mass spectrometry, and the bands identified as Clp or co-chaperonin subunits are marked (Fig. 1A) (Supplementary Dataset 1). Though the migration pattern of Clp subunits was slightly different from the previous report due to difference in protein preparation and SDS-PAGE conditions, the same major Clp subunits were identified, except for ClpT3 (Supplementary Table 1) ${ }^{32}$. In addition to the Clp subunits, the three co-chaperonin proteins Cpn11, Cpn20, and Cpn23 were identified with notable abundance (Supplementary Table 1)(Supplementary Dataset 1). The presence of clpP1 gene products $\mathrm{ClpP} 1_{\mathrm{H}}, \mathrm{ClpP} 1_{\mathrm{C}}$ and $\mathrm{ClpP} 1_{\mathrm{C}}$, and of $\mathrm{Cpn} 20$ in the purified $\mathrm{ClpP}$ complex was confirmed by immunoblotting using antisera against the Strep-tag and against Cpn20 (Fig. 1C). It is of note that the two bands detected for both $\mathrm{ClpP} 1_{\mathrm{C}}$ and $\mathrm{ClpP} 1_{\mathrm{C}}$, might result from the differentiated peptidase processing. To confirm the interaction of the Clp complex with the co-chaperonins, we performed a co-immunoprecipitation 
experiment using Cpn20 antiserum. As shown in Fig. 1D, Clp subunits ClpP1C, ClpP4, ClpR6, and ClpT4 were detected in the immunoprecipitate by immunoblotting. The interaction between the ClpP complex and chaperonins in vitro was further validated by size exclusion chromatography (Fig. 1E). Some of recombinantly produced Cpn20 co-migrated with the Chlamydomonas $\mathrm{ClpP}$ complex, and co-migration was even more marked with a mixture of the three co-chaperonins Cpn11/20/23. Note that Cpn20 was also detected after long time exposure in the $\mathrm{ClpP}$-only fractions (CrClpP row) because the co-chaperonin complex co-purifies with endogenous ClpP. No co-migration of Cpn subunits was observed with the E. coli $\mathrm{ClpP}$ complex, and the E. coli co-chaperonin GroES co-migrated neither with Chlamydomonas nor E.coli ClpP. These results indicate that the interaction between the co-chaperonin and the ClpP complex can be reconstituted in vitro and is a specific feature of the chloroplast system. This conclusion is in line with the finding that Arabidopsis Cpn20 interacts with the Arabidopsis Clp complex in vivo ${ }^{20}$.

Beta-casein is a commonly used model substrate for the Clp protease (CaseinoLytic Peptidase) ${ }^{33,34}$. To analyze protease activities of purified ClpP complexes, we performed protein degradation assays. ClpP of E.coli (EcClpP) could not degrade $\beta$ casein, unless $4 \mu \mathrm{M}$ of the ClpP activator acyldepsipeptide (ADEP) was present in the reaction (Figs. 2A, 2B and Extended Data Fig. 2A). ADEP increases the interaction between ClpP monomers, competes with the Clp ATPases for their binding sites on ClpP, and triggers a closed- to open-gate transition of the substrate entrance pore, which is otherwise tightly closed ${ }^{35,36}$. In contrast, purified Chlamydomonas ClpP was able to degrade $\beta$-casein without need for ADEP. ADEP slightly accelerated protein degradation by CrClpP, but only at a high concentration of $18 \mu \mathrm{M}$, not of 4 or $8 \mu \mathrm{M}$ (Figs. 2A, 2B and Extended Data Fig. 2A). Given the specific interaction between Chlamydomonas $\mathrm{ClpP}$ and the co-chaperonin complex (Fig. 1), we wondered whether co-chaperonins had an effect on ClpP proteolytic activity. Recombinant co-chaperonins Cpn20 and Cpn11/20/23 both slowed down the proteolytic activity of CrClpP (Figs. 2C and 2D), but co-chaperonin GroES had no effect (Extended Data Figs. 2A and 2B). This 
inhibitory effect was overcome by the addition of $18 \mu \mathrm{M}$ ADEP (Figs. 2C and 2D). CrClpP hydrolyzed $\beta$-casein into several fragments of lower molecular mass ranging from 15 to $20 \mathrm{kDa}$, which were not observed with EcClpP (Extended Data Fig. 2A). Hence, casein degradation by $\mathrm{CrClpP}$ appeared less processive than by $\mathrm{EcClpP}+\mathrm{ADEP}$. Moreover, Cpn20 was still bound to the CrClpP complex after incubation with $18 \mu \mathrm{M}$ ADEP (Extended Data Fig. 2C), indicating that either ADEP and the co-chaperonin bind to different sites on the ClpP core complex or ADEP cannot expel the cochaperonin from its binding sites. As tiny amounts of AAA+ chaperones might have co-purified with $\mathrm{CrClpP}$, we cannot conclude that $\mathrm{CrClpP}$ is proteolytically active in the complete absence of AAA+ chaperones. However, treatment with hexokinase/glucose, which will remove all ATP from the buffer, had no effect on casein degradation (Extended Data Fig. 2A), suggesting that CrClpP can degrade this substrate in a completely energy-independent process.

\section{Cryo-EM structure of ClpP complex and subunit assignment}

To analyze structural features of the Chlamydomonas plastidic ClpP complex, we performed cryo-EM single particle analysis on the system. Purified ClpP complexes were applied to specimen grids and vitrified for cryo-EM analysis (Extended Data Fig. 3 and 4). After 3D classification and iterative refinement, the optimally visualized particle groups were selected to generate two major groups, named ClpP-S1 and ClpPS2, at resolutions of $3.3 \AA$ and $3.6 \AA$, respectively. The main difference between these two groups was the appearance of a small cap in the latter particles (Figs. 3A-B and Extended Data Figs. 3C, 4A and 4D). Focused 3D classification, particle re-extraction, re-centering and refinement of the cap, which was later identified as co-chaperonin Cpn11/20/23, generated particles with better structural features and more complete mobile loops at $4.8 \AA$ A resolution (Extended Data Figs. 3C and 4B). We further combined the $4.8 \AA \mathrm{Cpn} 11 / 20 / 23$ and ClpP-S2 maps using the vop maximum command in Chimera and generated a ClpP-S2-composite map (ClpP-S2c)(Fig. 3B and Extended Data Fig. 3C). The ClpP-S1 map displayed an obviously asymmetric structural conformation with a height of about $108 \AA$, a width of about $105 \AA$ and a length of 
about $148 \AA$. The diameters of the central pore of ClpP-S1 are $28 \AA$ and $20 \AA$ for the top and bottom rings in the cut away views, respectively (Fig. 3A). This difference in pore size implied that there may exist a functional division between top and bottom ring in terms of substrate admittance. The cap in the ClpP-S2 particles increased the height to about $150 \AA$ and the width to $118 \AA$, while the length was the same as ClpP-S1 (Figs. $3 \mathrm{~A}$ and $3 \mathrm{~B})$. Overall, the resolution in the middle of the particles was higher than on the surface (Extended Data Fig. 4D), especially for ClpP-S1 where the resolution in the particle core reached about $3 \AA$, which allows the identification of amino acid side chains.

To analyze the structural features of the CrClpP complex, the crystal structure of the E.coli ClpP complex (PDB: 1TYF) was manually docked into the CrClp-S1 density map (Fig. 3C) ${ }^{13}$. The EcClpP structure fits well into the ClpP-S1 map around the central pore, with clear separation of double rings. Compared to the symmetrical EcClpP structure, three prominent additional densities appeared in ClpP-S1 that were labeled respectively as A1, A2 and A3. The A1 density is very large and locates adjacent to the interface of the two rings like a handle, while densities A2 and A3 appear to be more similar and locate to the peripheral region of the top ring.

In Chlamydomonas, three P-type (ClpP1, P4, P5), five R-type (ClpR1-4, R6) and two T-type $(\mathrm{ClpT} 3, \mathrm{~T} 4)$ subunits constitute the $\mathrm{ClpP}$ complex. $\mathrm{ClpP} 1_{\mathrm{H}}$ is processed by unknown peptidases to generate $\mathrm{ClpP} 1_{\mathrm{N}}, \mathrm{ClpP} 1_{\mathrm{C}}$ and $\mathrm{ClpP} 1_{\mathrm{C}}$, subunits, which are part of the ClpP core complex. An alignment of amino acid sequences of P- and R-type subunits with EcClpP revealed a conserved region corresponding roughly to amino acids 27-175 in EcClpP (Extended Data Fig. 5), which served to build the structures of the P- and R-type subunits (Extended Data Fig. 6A). The structure of ClpP1 $1_{\mathrm{N}}$ could not be built after many trials. To assign the subunits into the ClpP core complex, the 14 subunits in the complex were designated as D1-D14 (Fig. 4A, left) and the individually built structures were manually fit into the central region of the ClpP-S1 map. Some specific sequences of $\mathrm{ClpP}$ and $\mathrm{ClpR}$ subunits were used for assigning them to the ClpPS1 map (Extended Data Fig. 5). Visualizations of map fitting of specific side chains 
allowed us to identify subunit locations in the Clp-S1 map (Extended Data Fig. 6B).

After subunit assignment into the ClpP-S1 map, the individual subunit structures were built manually with continuous map densities. The structures of the $\mathrm{N}$ - and $\mathrm{C}$ termini of some subunits could not be solved due to discontinuity in the densities. Information on the resolved sequences is summarized in Supplementary Table 2. The superimposition of the individual subunit structure onto its corresponding electron density indicates that the models fit well into the corresponding maps (Extended Data Fig. 7A). Overall, we could assign one ClpR6, three ClpP4, and three ClpP5 subunits to the top ring (designated as P-ring), and one of each ClpR1-4 and three $\mathrm{ClpP} 1_{\mathrm{C}}$ subunits to the bottom ring (Figs. 4A-C). Values of around 0.7 in the correlation coefficient (CC) chart corroborate our model-to-map fitting for each subunit (Extended Data Fig. 7B). Still, some regions in the ClpP structure could not be solved, as shown by white regions in the core complex map density (Fig. 4B and Extended Data Fig. 7C). Notice that no subunits or sequences could be fit into the additional A2 and A3 maps (Extended Data Fig. 7C), including the long C-terminal sequences (Val196-Trp296) of the adjacent ClpP4 subunit, which remains unsolved. Conversely, no density could be ascribed to the large IS1 sequence characteristic of $\mathrm{ClpP} 1_{\mathrm{H}}{ }^{24,26}$. The T4 subunit could be assigned to the A1 density, which is located adjacent to the interface of the two rings (Figs. 4B-C). The additional A1 map density, located at the interface of the two rings, is formed by a very long helix originating from the C-terminus of the ClpR3 subunit in the bottom ring, as well as by the T4 subunit and several amino acids from the ClpR6 subunit in the top ring (Fig. 4D). Some unassigned map density in A1 might be contributed by the C-terminus of ClpR3, and by $\mathrm{ClpT} 3$ or $\mathrm{ClpP} 1_{\mathrm{N}}$ subunits. The $\mathrm{A} 1$ region connects the two rings like a handle and can be assumed to stabilize the ClpP core complex. Moreover, the $\mathrm{C}$-terminus of $\mathrm{ClpR} 4$ protrudes into a region next to the ClpP5 subunits in the top ring, and this might contribute to stabilize the core complex, as well (Fig. 4E).

The electrostatic potential is not equally distributed around the central pores of the two rings through which substrates enter the catalytic chamber, suggesting a differential 
affinity to substrates (Fig. 4F). The AAA+ chaperones interacts with EcClpP by protruding their flexible IGF loops into the hydrophobic clefts of $\mathrm{ClpP}$ which are formed at the interface of two subunits ${ }^{16}$. Similarly, seven hydrophobic clefts were found to form at the surface of the top ring of ClpP by amino acids from two adjacent subunits. These hydrophobic clefts are arranged in a circular manner with seven-fold symmetry and were observed only on the top ring (Fig. 4G). However, we cannot completely rule out that similar hydrophobic clefts exist on the bottom ring, because the models of the three ClpP1c subunits in the bottom ring are not complete. A schematic model of the overall structure of the ClpP core complex with assigned subunits is shown in Fig. 4A.

\section{The cap on the top of the ClpP core is the co-chaperonin}

We have shown that the co-chaperonin complex interacts with the ClpP core in vivo and in vitro (Fig. 1). Previous work from us and others showed that the authentic cochaperonin complex in vivo consists of two Cpn20 subunits, one Cpn23 subunit, and one Cpn11 subunit ${ }^{37,38}$. Compared to Clp-S1, the ClpP-S2c map showed a dome-like density located on the top of the ClpP core complexes (Fig. 3, labeled A4 in Extended Data Fig. 8A) which we attributed to the co-chaperonin. Since Cpn20 and Cpn23 each have two GroES-like domains, we split them into Cpn20-N, Cpn20-C, Cpn23-N, and Cpn23-C regions after cleavage of transit peptide ${ }^{39}$ and performed a sequence alignment with Cpn11 and GroES (Fig. 5A). Sequences contributing to the roof of the dome-shaped co-chaperonin complex were present in GroES, Cpn23-N, Cpn23-C and Cpn20-C. This sequence was much shorter in Cpn11 and missing in Cpn20-N. Based on these specific roof characteristics we could assign Cpn11, Cpn23, and two Cpn20 subunits (Cpn20-1 and Cpn20-2) to the co-chaperonin map (Fig. 5B). The CC chart indicates a good model-to-map fitting for both $\mathrm{Clp}$ and co-chaperonin subunits (Extended Data Fig. 8B) and the superimposition of the individual co-chaperonin subunit structure onto its corresponding electron density indicates that the models fit well into the corresponding maps (Extended Data Fig. 8C). We further refined the model and fit five of the seven mobile loops into the densities that extended from the 
bottom of the co-chaperonin dome (belonging to the Cpn20 and Cpn11 subunits, Fig. 5B, left) (Fig. 5B, left). No densities were observed for the loops of the Cpn23 subunit, although the sequences are conserved (Fig. 5A).

Inspection of the map revealed that the co-chaperonin cap is tilted by about $4^{\circ}$ relative to the $\mathrm{ClpP}$ symmetry axis (Figs. 5C and 5F). This tilt increases the distance between $\mathrm{ClpP}$ and $\mathrm{Cpn} 23$ versus $\mathrm{ClpP}$ and $\mathrm{Cpn} 20$, suggesting an intimate interaction at the Cpn20 side. A similar interaction, but with a tilt angle of $11^{\circ}$, has been observed for the asymmetrical EcClpP with its $\mathrm{AAA}+$ chaperone $\mathrm{ClpX}{ }^{16}$. While that interaction is accompanied by a symmetry mismatch, this is not the case for the interaction between co-chaperonin and ClpP, which share a 7-fold symmetry. In E.coli, the IGF loops of $\mathrm{ClpX}$ insert into hydrophobic clefts located at the surface of $\mathrm{EcClpP}^{15}$. Close inspection of the density map of the ClpP core complex in ClpP-S2c clearly revealed five extra densities in the hydrophobic clefts of the top ClpP ring (red and orange in Fig. 5D). Three of these densities were confidently identified as mobile loops stemming from Cpn11, contacting the ClpP5/R6 interface, and Cpn20-1C and Cpn20-2C, both contacting a ClpP5/P4 interface (red in Fig. 5E). The mobile loops from Cpn20-1N and Cpn20-2N might contact ClpP clefts as well, but at a slightly outward location, which could account for two more extra densities observed in clefts of the top ClpP ring (orange in Fig. 5D; Extended Data Fig. 8D). An assignment of the densities in the two cleft regions was complicated by the close-by unassigned A2/A3 densities. For the two remaining hydrophobic clefts, in register with Cpn23, no extra map densities were observed (broken red lines in Fig. 5D), indicating that there is no direct interaction between Cpn23 and the ClpP core. In summary, the co-chaperonin appears to interact with the ClpP core by inserting at least three mobile loops into hydrophobic clefts on the surface of the top ring of ClpP (Fig. 5F), similar to the interaction of EcClpP with its AAA+ chaperone ClpX. Notice that the same loops of the co-chaperonin mediate its interaction with the Cpn60 chaperonin ${ }^{6}$. The interactions of the co-chaperonin with

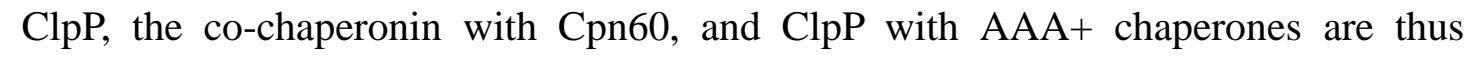
mutually exclusive. 


\section{DISCUSSION}

\section{The chloroplast co-chaperonin has multiple functions}

320 Many lines of evidence indicate that Cpn20 functions as a co-chaperonin for Cpn60 to 321 assist protein folding in chloroplasts ${ }^{38,40-42}$. However, Cpn20 appears to exhibit 322 functions independent of the Cpn60 chaperonin: in Arabidopsis, Cpn20 has been shown 323 to play roles in abscisic acid (ABA) signaling and the activation of iron superoxide 324 dismutase (FeSOD) ${ }^{7-9}$. Here we show that a fraction of the Chlamydomonas chloroplast 325 Cpn11/20/23 co-chaperonin complex co-purified with the ClpP core complex, and this 326 interaction was robust enough to withstand several chromatography purification steps 327 (Fig. 1). This interaction was shown before in Arabidopsis ${ }^{20}$. The complexes between 328 co-chaperonin and ClpP were confirmed by cryo-EM and in vitro reconstitution, and 329 the co-chaperonin was shown to slow down the proteolytic activity of chloroplast ClpP 330 (Figs. 2B and 5). The co-chaperonin caps the top-ring of the ClpP complex like a dome, similar to its interaction with Cpn60. Thus, the co-chaperonin might inhibit ClpP activity by blocking the entrance of unfolded proteins into the central cavity of the ClpP core complex via the pore of the top ring. The co-chaperonin complex interacts with $\mathrm{ClpP}$ and the Cpn60 chaperonin via the same mobile loops extending from the bottom of the dome (Fig. 5E). Therefore, the Cpn20/23/11 co-chaperonin complex appears to play a dual role in chloroplast protein quality control. Since its interaction with the

337 Cpn60 chaperonin contributes to protein folding, the binding of the co-chaperonin to 338 ClpP would simultaneously limit the efficiency of protein folding and inactivate a substantial part of the chloroplast's degradation capacity. Releasing the co-chaperonin 340 from ClpP would activate both activities at the same time, which might be important upon rapid challenges of proteostasis by environmental changes. In future studies, it 342 will be interesting to quantify the relative stoichiometry of the chaperonin, co343 chaperonin, and ClpP complexes in the Chlamydomonas chloroplast and their 344 interactions under various growth or stress conditions. It is of note that the very abundant RbcL subunit of Rubisco is a substrate for both chaperonin and ClpP, and that 
translational attenuation of $c l p P l$ is lethal in the presence of $r b c L$ mutations preventing its folding ${ }^{27}$. While we initially postulated that the lack of sufficient ClpP would cause poisoning of the chaperonin by an unfoldable substrate, we must now also consider an additional hypothesis, that the mutant RbcL, by increasing the residence time of the cochaperonin on and ever-busy Cpn60, would deregulate ClpP proteolysis.

The fact that the three Cpn60 subunits were also detected by mass spectrometry in our preparation (Supplementary Dataset 1) raises another possibility, namely that ClpP and Cpn60 interact to regulate chloroplast protein homeostasis. Non-foldable substrate proteins, released from the chaperonin, need to be recognized by Clp to be degraded to prevent poisoning of the chaperonin. The co-chaperonin, by maintaining its interaction with the non-foldable substrate, could help direct it to the protease. In line with this idea, a cooperation between Trigger Factor, an ATP-independent ribosome-associated chaperone, and ClpXP was recently reported to promote the degradation of some substrates ${ }^{43}$.

\section{Molecular architecture of chloroplast ClpP}

The first structure of the E. coli ClpP core complex, obtained by X-ray crystallography, revealed 14 identical subunits in two stacked rings ${ }^{13}$. The ClpP structures solved afterwards all consisted of a single or two types of ClpP subunits. In contrast, the chloroplast ClpP core complex solved here combines 10 different subunits, either of the P-type (harboring a functional catalytic site) or R-type (lacking catalytic residues) or T-type (homologous to the N-domain of AAA+ chaperones), distributed with uneven stoichiometry between two rings of different subunit composition. The subunit composition of the two rings of plastid ClpP from Arabidopsis was reported previously by the van Wijk and Clarke groups $20,22,30,44,45$. The designated P-ring contains ClpP3, 4, 5 and 6 in a 1:2:3:1 ratio, while the designated R-ring consists of ClpP1 and ClpR1, 2, 3 and 4 in a 3:1:1:1:1 ratio. It was suggested that the two rings might exhibit different proteolytic capacities because only three catalytic subunits are present in the R-ring compared to seven in the P-ring. In the Chlamydomonas $\mathrm{ClpP}$ core complex reported here, the top ring consists of three $\mathrm{ClpP} 4$, three ClpP5 and one ClpR6 subunits. Because 
algal ClpP4 forms a sister clade to land plant ClpP3/ClpP4, and ClpR6 is clearly derived from ClpP6 by loss of a catalytic residue ${ }^{32}$, the top ring of Chlamydomonas is clearly homologous to the Arabidopsis P-ring. Similarly, the bottom ring, with three ClpP1c and one each of ClpR1, 2, 3, and 4 subunits (Fig. 4C) can be called the R-ring. Because the diameter of the protein entrance pore in the P-ring is much larger than that in the Rring (Fig. 3A), we propose that the former is the functional entry site of substrates. Only the P-ring was observed to interact with the co-chaperonin (Fig. 5), in line with a central regulatory role for this interaction. Our structure supports the notion that substrates are degraded inside a unique central cavity by a variety of active sites, possibly showing distinct chemical specificities as in the proteasome ${ }^{46}$. Each subunit within each ring may also be functionally unique in terms of substrate selection, delivery and unfolding, and of interaction with the co-chaperonin. A good example is the specific interactions of the ClpP4/5 clefts with Cpn20 subunits (Fig. 5E, Extended Data Fig. 8D).

Interesting differences can be noted between algal and land plant ClpP. With only 6 active subunits in its P-ring, the Chlamydomonas enzyme is slightly less asymmetrical than that of land plants in terms of catalysis (both have 3 catalytic sites in the R-ring). Importantly, the inactive ClpR6 subunit of the P-ring contributes, together with two other alga-specific features, namely the C-terminal extension of the R-ring ClpR3 and ClpT4, to the A1 side density that connects the two rings. In land plants, T-type Clp subunits were suggested to stabilize the ClpP core by interacting with both rings ${ }^{20,21}$, and it will be interesting to see if they form a structure similar to the A1 side-mass. Note however that the Chlamydomonas ClpT3/4 are vastly different from the ClpT1/2 of land plants ${ }^{32}$, to the extent that they may not even be orthologous. Given that the $\mathrm{N}$ terminal domain of AAA+ chaperones, to which ClpTs are homologous, play a role in substrate selection, the possibility arises that their dual structural/functional role was acquired several times in the evolution of the plastid Clp system. Furthermore, the Cterminus of ClpR4 protrudes to the P-ring, which also might stabilize the ClpP core complex. The massive contacts between the two rings may explain the high stability of the ClpP core, exemplified by our observation that the two-rings could not be separated 
404 by high salt treatment, in contrast to its homologs in E. coli and Arabidopsis plastids ${ }^{45}$, $405 \quad 47$.

406 Proteolytic activity of the ClpP is regulated by the co-chaperonin

407 Structures of several different AAA+ chaperones with their ClpP cores have emerged 408 recently, providing detailed information on the six-seven symmetry mismatch between 409 the two complexes, and how it impacts their interaction dynamics ${ }^{15,16,18}$. ClpX is tilted 410 by $11^{\circ}$ without major conformational changes upon binding to ClpP. As a result, the 411 symmetry axes of the protease and the AAA+ chaperone are not aligned so that the 412 translocation pathway for unfolded peptides is not straight but twisted. We also found 413 a tilt of $4^{\circ}$ between the axes of the co-chaperonin and the ClpP core (Fig. 5C). However, 414 the fact that a single type of particle was obtained, with specific interactions between 415 co-chaperonin and Clp subunits, together with the absence of a symmetry mismatch, 416 suggest that the two complexes do not rotate and that the co-chaperonin instead operates 417 like a cap stably sealing the entrance to the ClpP core.

418 Because none of the three known chloroplast AAA+ chaperones (ClpC1, ClpD1, $419 \mathrm{ClpB} 3$ ) were co-purified with $\mathrm{ClpP}$ in this and previous studies, they might not 420 cooperate with $\mathrm{ClpP}$ in the chloroplast as they do in bacteria and mitochondria. Since 421 the purified ClpP core complex was able to degrade the model substrate casein in vitro 422 with no need for addition of ADEP, it appears possible that chloroplast ClpP can 423 degrade proteins in vivo without the assistance of any type of chaperone. Regulation of 424 protease activity may be carried out by the co-chaperonin or by small-molecule 425 activators acting like ADEP.

427 METHODS

428 Construction of expression plasmids

429 The $C l p P$ gene sequence from $E$. coli was amplified by PCR on genomic DNA and 430 cloned into the pHUE vector with restriction enzymes to generate $\mathrm{EcClp}$-pHUE. The 431 produced EcClp protein contains ubiquitin and a $6 \times \mathrm{His}$ tag at its $\mathrm{N}$-terminus that 432 facilitated subsequent protein affinity purification. The forward and reverse primers 
435 BamHI and HindIII restriction sites, respectively. The construction of the cochaperonin expression plasmids, GroES-pET11a, CrCPN20-pQlinkT and CrCPN11/ 20/23-pQlinkT, were described earlier ${ }^{37}$.

\section{Chlamydomonas reinhardtii strains and growth conditions}

Chlamydomonas strain ClpP1-strep (\#8), used for the purification of the Clp complex via Strep-tag affinity purification, has been describe previously ${ }^{26}$ and is freely available upon request. The strain was kept on solid TAP medium containing $100 \mu \mathrm{g} / \mathrm{ml}$ of spectinomycin at $25^{\circ} \mathrm{C}$ under continuous illumination $\left(40 \mu \mathrm{mol}\right.$ photo $\left.\mathrm{m}^{-2} \mathrm{~s}^{-1}\right)$ or a photoperiod rhythm (12 h light/12 h dark).

\section{Purification of the ClpP complex from $C$. reinhardtii}

Purification of the ClpP complex from Chlamydomonas reinhardtii was conducted as described previously with some modifications ${ }^{48}$. The ClpP1-strep strain was inoculated into $24 \mathrm{~L}$ TAP liquid medium for 4 days under continuous light $\left(40 \mu \mathrm{mol}\right.$ photo $\mathrm{m}^{-2} \mathrm{~s}^{-}$ $\left.{ }^{1}\right)$. When cell numbers reached around $6 \times 10^{6}$ cells $/ \mathrm{mL}$, the cells were collected by centrifugation at $3600 \mathrm{~g}$ for $6 \mathrm{~min}$ and resuspended in buffer A (20 mM Tris- $\mathrm{HCl} \mathrm{pH}$ $8.0,150 \mathrm{mM} \mathrm{NaCl}$ ). The volume of the cell slurry was adjusted to $200 \mathrm{ml}$ by the addition of $1 \mathrm{mM}$ EDTA, $1 \mathrm{mM}$ PMSF, and two tablets of EDTA-free protease inhibitor cocktail (Roche). Unless otherwise stated, subsequent steps were performed at $4{ }^{\circ} \mathrm{C}$. The cells were sonicated and centrifuged at $36,000 \mathrm{~g}$ for $30 \mathrm{~min}$ to remove debris. $1 \mathrm{mg} / \mathrm{L}$ avidin and $6 \mathrm{mM} \mathrm{MgCl} 2$ were added to the supernatant to improve the Strep tag binding efficiency with Strep-Tactin beads (Novagen). Next, the supernatant was further clarified by ultracentrifugation at $150,000 \mathrm{~g}$ for $1 \mathrm{~h}$ (Beckman Coulter rotor, 70Ti). Ammonium sulfate was slowly added to the supernatant at $25 \%$ saturation. After gently stirring for $30 \mathrm{~min}$, the produced aggregates were removed by centrifugation at 36000 g for 20 min. Buffer E (20 mM Tris-HCl pH 8.0, 1 mM DTT, 10\% glycerol) was added to the supernatant to dilute the ammonium sulfate concentration to $0.5 \mathrm{M}$ and the solution was applied to pre-equilibrated hydrophobic column after passing through a 
$0.22 \mu \mathrm{m}$ filter (Hitrap phenyl, GE Healthcare). Proteins were eluted by an ammonium sulfate gradient from $0.5 \mathrm{M}$ to $0 \mathrm{M}$ with ten column volumes. Clp-containing fractions were collected after visualization with SDS-PAGE and transferred to a Strep-Tactin gravity column which was pre-equilibrated with buffer B (20 mM Tris-HCl pH 8.0, 100 $\mathrm{mM} \mathrm{NaCl}, 1 \mathrm{mM}$ DTT). The protein was eluted with buffer $\mathrm{C}$ (buffer $\mathrm{B}+10 \%$ glycerol+2.5 mM Desthiobiotin (Novagen)) in four column volumes. The ClpP complex was concentrated and subjected to a Superdex-200 column pre-equilibrated with buffer D (20 mM Tris- $\mathrm{HCl} \mathrm{pH} 8.0,80 \mathrm{mM} \mathrm{NaCl}, 10 \%$ glycerol) and the desired fractions were collected. Purified ClpP complexes were concentrated to $\sim 2 \mathrm{mg} / \mathrm{ml}$ by Amicon Ultra-15 Centrifugal Filter Units (Merck Millipore, Beijing China) with 100 $\mathrm{kDa}$ cut-off, supplemented with $10 \%$ glycerol and frozen at $-80^{\circ} \mathrm{C}$.

\section{Purification of recombinantly expressed proteins}

EcClpP The EcClp-pHUE plasmid was transformed into the E. coli BL21 (DE3) strain, and then transferred to $4 \mathrm{~L}$ lysogeny broth (LB) medium containing $100 \mu \mathrm{g} / \mathrm{L}$ ampicillin. When E.coli was grown to an OD 600 of $\sim 0.6,1 \mathrm{mM}$ isopropyl $\beta$-D-1thiogalactopyranoside (IPTG) was added to induce protein expression. After 4 hours, cells were collected by centrifugation at $4000 \mathrm{~g}$. Unless otherwise stated, all purification steps were performed at $4{ }^{\circ} \mathrm{C}$. Cells were resuspended in lysis buffer $(20 \mathrm{mM}$ Tris- $\mathrm{HCl}$, pH 8.0, $300 \mathrm{mM} \mathrm{NaCl}, 1 \mathrm{mM}$ DTT, $10 \mathrm{mM}$ imidazole, $1 \mathrm{mM}$ phenylmethylsulfonyl fluoride (PMSF)) and lysed by sonication. Debris was removed by centrifugation at $36000 \mathrm{~g}$ for $40 \mathrm{~min}$ and the supernatant was passed through a $0.22 \mu \mathrm{m}$ filter followed by transferring into the Ni-NTA gravity column. Protein was eluted with elution buffer (20 mM Tris- $\mathrm{HCl}, \mathrm{pH} 8.0,300 \mathrm{mM} \mathrm{NaCl}, 1 \mathrm{mM}$ DTT, $250 \mathrm{mM}$ imidazole) after two washes with buffer (20 mM Tris-HCl, pH 8.0, $300 \mathrm{mM} \mathrm{NaCl}, 1 \mathrm{mM}$ DTT, $25 \mathrm{mM}$ imidazole). Proteins in the eluted fractions were collected and digested with deubiquitinating enzyme USP2cc (1:50 molar ratio) to remove the ubiquitin-6xHis tag. The digested solution was applied to Ni-NTA column again and the flow through was collected which contained EcClpP protein without tag. The collected protein was concentrated using Amicon Ultra-15 Centrifugal Filter Units (Merck Millipore, Beijing 
China) with $100 \mathrm{kDa}$ cut off and frozen at $-80^{\circ} \mathrm{C}$.

492 GroES, Cpn20 and Cpn11/20/23. All co-chaperonins were purified using the same

493 method. Individual expression plasmid GroES-pET11a, Cpn20-pQlinkT or 494 Cpn11/20/23-pQlinkT was transformed into E. coli strain BL21 (DE3). Transformants 495 were picked and transferred to $6 \mathrm{~L}$ of LB medium containing $100 \mu \mathrm{g} / \mathrm{L}$ ampicillin. 496 When cells were grown to an OD 600 of $\sim 0.6,1 \mathrm{mM}$ IPTG was added to induce protein 497 expression at $37^{\circ} \mathrm{C}$ for 4 hours. Cells were harvested by centrifugation at $3600 \mathrm{~g}$ for 6 min. Unless otherwise stated, all subsequent steps were performed at $4^{\circ} \mathrm{C}$. Cell pellets were resuspended in lysis buffer (30 mM Tris-HCl, pH 8.0, $60 \mathrm{mM} \mathrm{NaCl}, 1 \mathrm{mM}$ DTT, $5001 \mathrm{mM}$ EDTA and $1 \mathrm{mM}$ PMSF) sonicated. Lysates were centrifuged at 36,000 $\mathrm{g}$ for 30 501 min to remove cell debris. The supernatant was passed through a $0.22 \mu \mathrm{m}$ filter followed

502 by transfer to a pre-equilibrated source 30Q column (GE Healthcare) with $1 \mathrm{ml} / \mathrm{min}$ 503 flow rate. Co-chaperonins were eluted by a linear salt gradient from $30 \mathrm{mM}$ to $1 \mathrm{M}$ $504 \mathrm{NaCl}$ with 10-fold column volume. Fractions containing protein according to UV 505 absorption were separated by $15 \%$ SDS-PAGE and visualized by Coomassie staining. 506 Eluted proteins were concentrated, then injected into a pre-equilibrated Superdex75 507 column (GE Healthcare) with a flow rate of $0.8 \mathrm{ml} / \mathrm{min}$. According to UV absorption, 508 the protein fraction was further analyzed with 15\% SDS-PAGE and Coomassie staining. 509 The pure co-chaperonin proteins were concentrated with Amicon Ultra-15 Centrifugal 510 filters with $30 \mathrm{kDa}$ cutoff, then flash-frozen in liquid nitrogen and stored at $-80^{\circ} \mathrm{C}$.

\section{$511 \quad$ Identification of CrClpP subunits by mass spectrometry}

512 The protein subunits of the CrClpP complex were separated on an SDS-PAGE gel and 513 visualized by Coomassie staining. Individual bands were cut out and digested by trypsin. 514 Liquid chromatography-mass spectrometry (LC-MS) was done on a Thermo Scientific 515 Q Exactive (QE) mass spectrometer at the Beijing Huada Protein R\&D Center Co., Ltd. 516 (Beijing, P.R.China). The Q Exactive mass spectrometry data were searched against the 517 Phytozome v12.1(Chlamydomonas reinhardtii) database and NCBI-Chlamydomonas 518 (taxid: 3052 ) database using $15 \mathrm{ppm}$ peptide mass tolerance and $20 \mathrm{~m} / \mathrm{z}$ fragment mass 519 tolerance. 


\section{Immunoprecipitation assays}

Protein A-sepharose beads coupled with $\mathrm{CrCpn} 20$ or strep-tag antibodies were preequilibrated in lysis buffer containing $20 \mathrm{mM}$ Hepes-KOH (pH7.5), $150 \mathrm{mM} \mathrm{NaCl}, 10$ $\mathrm{mM} \mathrm{MgCl} 2,20 \mathrm{mM} \mathrm{KCl}$ and $2 \mathrm{mM}$ EDTA, then incubated with total protein or stroma protein which was prepared with the same lysis buffer under gentle stirring at $4^{\circ} \mathrm{C}$. Protein A beads were washed three times with lysis buffer containing 0.1\% Tween 20 . Bound protein complexes were eluted with $2 \%$ SDS for $1 \mathrm{~h}$ at $4{ }^{\circ} \mathrm{C}$. The eluted proteins were separated by $12 \%$ SDS-PAGE and analyzed by immunoblotting with serum in $1: 3000$ dilution.

\section{Analytical gel filtration}

The ClpP interaction with co-chaperonins was analyzed by analytical gel filtration as described previously with some modifications ${ }^{38} .1 \mu \mathrm{M}$ ClpP and $2 \mu \mathrm{M}$ co-chaperonin were incubated for $30 \mathrm{~min}$ at $4^{\circ} \mathrm{C}$ in $20 \mathrm{mM}$ MOPS-KOH, pH 7.5, $80 \mathrm{mM} \mathrm{NaCl}, 10$ $\mathrm{mM} \mathrm{MgCl} 2,10 \mathrm{mM} \mathrm{KCl}, 1 \mathrm{mM}$ DTT, $10 \%$ glycerol. Then the protein complexes were loaded onto a Superdex 200 PC 3.2/10 column (GE Healthcare) at a flow rate of 0.05 $\mathrm{ml} / \mathrm{min} .50 \mu \mathrm{L}$ fractions were collected and analyzed by immunoblotting with Strep-tag and Cpn20 antibodies.

\section{Asymmetric flow field-flow fractionation with multi-angle light scattering}

\section{(AFFFF-MALS)}

$50 \mu \mathrm{g}$ protein of purified CrClpP were loaded into a AFFFF-MALS device with a flow rate of $0.8 \mathrm{ml} / \mathrm{min}$ and a cross-flow rate of $2 \mathrm{ml} / \mathrm{min}$ using a $350 \mathrm{~mm}$ spacer and $10 \mathrm{kDa}$ RC membrane (Wyatt Technology, Santa Barbara, CA, USA). The monitor methods employed a multiple-angle light scattering detector (DAWN HELEOS II, 658 nm; Wyatt Technology), a UV detector (1100 series, 280 nm; Agilent Technologies In., Santa Clara, CA, USA) and a differential refractive index detector (Optilab rEX, 658 nm; Wyatt Technology) ${ }^{49}$. The CrClpP molecular weight was calculated in the presence of $\mathrm{Dn} / \mathrm{dc}$ values equal to $0.185 \mathrm{ml} / \mathrm{g}$.

\section{$\beta$-casein degradation assays}

Degradation of the substrate protein $\beta$-casein was visualized by Coomassie Blue $R$ 
staining after separating proteins via SDS-PAGE (12\% acrylamide). The reaction mixture contained $20 \mathrm{mM}$ Tris- $\mathrm{HCl} \mathrm{pH} 8.0,120 \mathrm{mM} \mathrm{NaCl}, 10 \mathrm{mM} \mathrm{KCl}, 10 \mathrm{mM} \mathrm{MgCl}_{2}$, $1 \mathrm{mM}$ DTT, $10 \%$ glycerol, $0.4 \mu \mathrm{M}$ EcClpP or CrClpP, $0.4 \mu \mathrm{M} \beta$-casein protein with or without $0.4 \mu \mathrm{M}$ co-chaperonin (GroES, Cpn20 and Cpn11/20/23). 4 to $18 \mu \mathrm{M}$ protease activator ADEP (\#sc-397312, Santa Cruz) dissolved in DMSO was added to the reaction as indicated. The reaction was performed at $30^{\circ} \mathrm{C}$, and the aliquots were taken at the indicated time points. The reaction was stopped by heating to $98^{\circ} \mathrm{C}$ for $10 \mathrm{~min}$. Each reaction was performed at least three times and densitometric quantification of $\beta$ casein from the reactions was made with Photoshop CS6.

\section{Cryo-EM sample preparation and data collection}

Holey carbon grids (Quantifoil R2/1, 200 mesh) were plasma cleaned using a Solarus plasma cleaner (Gatan), and an aliquote of $2 \mu \mathrm{CrClpP}$ sample was placed onto the glow-discharged grid. Then the grid was flash-frozen in liquid ethane by a Vitrobot Mark IV (Thermo Fisher Scientific). Movies were taken on a Titan Krios transmission electron microscope (Thermo Fisher Scientific) equipped with a Cs corrector and operated at an accelerating voltage of $300 \mathrm{kV}$ with a nominal magnification of $18,000 \mathrm{x}$ (Supplementary Table 3). Movies were collected by using a K2 Summit direct electron detector (Gatan) in super-resolution mode (yielding a pixel size of $1.318 \AA$ after 2 times binning). Each movie was dose-fractioned into 38 frames and the exposure time was $7.6 \mathrm{~s}$ with $0.2 \mathrm{~s}$ for each frame, producing a total dose of $\sim 38 \mathrm{e}^{-} / \AA^{2}$. The defocus value of the data set varied from -0.8 to $-2.5 \mu \mathrm{m}$. We employed the SerialEM automated data collection software package to collect the images ${ }^{50}$.

\section{Image processing and 3D reconstruction}

A total of 8,064 movies were applied for $\mathrm{CrClpP}$ structure determination. Unless otherwise specified, single-particle analysis was mainly executed in RELION 3.1 $1^{51,52}$. All images were aligned and summed using MotionCorr $2{ }^{53}$ and CTF parameters were determined using CTFFIND4 ${ }^{51,52}$. We obtained 1,351,977 particles by automatic particle picking followed by manual checking, and 578,978 particles remained for further processing after reference-free $2 \mathrm{D}$ classification. Through one round of 3D 
classification, a ClpP-S1 dataset of 306,743 particles and a CrClpP-S2 dataset of 134,904 particles were obtained. Then multiple rounds of reference free 2D and 3D classification were applied to clean up the particles for each dataset. Classes with better structural features were combined and yielded ClpP-S1 dataset consisting 131,245 particles. After further Bayesian polishing and CTF refinement, a map at $3.3 \AA$ resolution was obtained. Two classes with better structural features were combined and yielded ClpP-S2 dataset consisting 49,759 particles. After Bayesian polishing and CTF refinement, a ClpP-S2 map at $3.6 \AA$ resolution was obtained. To improve the local resolution of the Cpn cap in the ClpP2-S2 map, the particles were subtracted by a soft mask focusing on the cap region and re-centered. We then applied 3D classification and obtained a cleaned-up dataset of 13,040 particles with better structural features especially more complete density of the Cpn mobile loops, which was further refined to $4.8 \AA$ resolution of the Cpn11/20/23 map. ClpP-S2 map was then combined with the Cpn11/20/23 map together by using the vopmaximum function in Chimera ${ }^{54}$, generating the composite ClpP-S2 map (termed ClpP-S2c). The overall resolution was determined based on the gold-standard criterion using an FSC of 0.143 . The local resolution estimation was determined by Local resolution function in RELION 3.1.

\section{Model building of Clp subunits with the co-chaperonin}

The structures of conserved regions of Clp subunits were predicted using the Rosetta server ${ }^{55,56}$. The predicted structures of conserved domains were docked rigidly into the density map in UCSF Chimera. The coordinates were further refined by the Real Space Refine module of the Phenix suite ${ }^{57}$. On this basis of refinement, the Rosetta enumerative sampling method was applied to build the remaining residues of each Clp subunit de novo ${ }^{56,58,59}$. The resulting model was adjusted manually in $\operatorname{Coot}^{60}$. About the co-chaperonin Cpn11/20/23 model, each Cpn11, Cpn20, Cpn23 homology model was built with the tFold server (Tencent AI Lab) or Rosetta server. These models were docked into the cryo-EM map of ClpP-S2c using the fit in map command in UCSF Chimera ${ }^{54}$. The resulting model was subjected to Rosetta and Phenix refinement ${ }^{57,56 .}$ The geometries and atomic model refinement statistics were evaluated by Molprobity 
in Phenix ${ }^{61}$.

Cryo-EM data acquisition, 3D process information and model refinement statistics are summarized in Supplementary Table 3. Figures were generated with either UCSF Chimera and ChimeraX ${ }^{54,62}$.

\section{Data availability}

Electron density maps have been deposited in the Electron Microscopy Data Bank under accession codes EMD-31171 for CrClpP-S1, EMD-31175 for ClpP-S2, EMD31173 for ClpP-S2c and EMD-31174 for Cpn11/20/23. Related atom coordinates file also has been submitted to the Protein Data Bank, with accession codes 7EKO for CrClpP-S1, and 7EKQ for CrClpP-S2c.

\section{Acknowledgments}

We are grateful to the staff of the NCPSS EM facility, Mass Spectrometry facility, and Database and Computing facility for instrument support and technical assistance. This work was funded by the Strategic Priority Research Program of Chinese Academy of Sciences (Grant No. XDA24020103-2, XDB37040103), the National Key Research and Development Program of China (2016YFD0100405, 2017YFA0503503) and the Ministry of Agriculture of China (2016ZX08009-003-005), the 'Initiative d'Excellence' program from the French State (Grant 'DYNAMO', ANR-11-LABX-0011-01), and the DFG (TRR 175, project C02). We thank Prof. Jean David Rochaix and Dr. Silvia Ramundo for their fruitful discussion.

\section{Author Contribution}

C. L. and Y.C. supervised the project. N. W. executed all biochemical experiments. Y. W. and X. Z. collected the cryo-EM data. Y. W. did data processing with initial map from X. Z. Y.W. and N.W. did model building and structural analysis. Q. Z. started the project and optimized the protein purification. C. P. performed the MS analysis. W. Z. and Y. L. helped to purify protein. O. V. and M. S. were involved in the project design, data analysis and interpretation. C. L., N. W., O. V. and M. S. wrote the manuscript with modification from Y. W. and Y. C.

\section{Competing Interests}


The authors declare that they have no competing interests.

Figure 1. Co-chaperonins interact with CrClpP

640

641

642

643

644

645

646

647

648

649

650

651

652

653

654

655

656

657

658

659

660

661

662

663

664

665

666

667

668

669

670

671

672

673

674

675

676

(A) Purified Chlamydomonas ClpP complexes were separated on 12-18\% SDSpolyacrylamide gels and visualized by Coomassie staining. Proteins in visible bands were identified by mass spectrometry and the bands identified as Clp or co-chaperonin subunits are marked. The gel is a representative of four independent experiments and mass spectrometry was performed twice with similar results.

(B) Purified ClpP and Cpn60 complexes were analyzed on a 5\% non-denaturing (ND) polyacrylamide gel and visualized with Coomassie staining. The arrowhead indicates the position of the oligomeric $820-\mathrm{kDa}$ Cpn60 complex and the asterisk the position of the $60-\mathrm{kDa}$ Cpn60 monomer. The gel is a representative of three independent experiments.

(C) Immunoblots analysis. Purified ClpP complexes were separated on a 15\% SDSpolyacrylamide gel, transferred to nitrocellulose and immunodecorated with anti-strep and anti-Cpn20 antibodies. The Strep-antibody recognized the three gene products of the plastid-encoded $c l p P$ gene, $\mathrm{ClpP} 1_{\mathrm{H}}, \mathrm{ClpP} 1_{\mathrm{C}}$ and $\mathrm{ClpP} 1_{\mathrm{C}}$. The experiment was repeated three times independently with similar results.

(D) Immunoprecipitation of Cpn20. Total cell lysates from the ClpP-Strep (\#8) strain (input) were incubated with protein A-Sepharose beads coupled to antibodies of either preimmune serum (pre) or anti-Cpn20 serum. Precipitated proteins were analyzed by immunoblotting using antisera against ClpP4, the Strep-tag (detecting ClpP1C), ClpR6, ClpT4 and Cpn60. The experiment was repeated three times independently with similar results.

(E) Co-migration of ClpP core complexes and co-chaperonins in size exclusion chromatography. $1 \mu \mathrm{M}$ ClpP protein complex was injected into a Superdex $200 \mathrm{PC}$ $3.2 / 10$ column after incubation with or without $2 \mu \mathrm{M}$ co-chaperonin for $30 \mathrm{~min}$ at $4^{\circ} \mathrm{C}$. Proteins were eluted with $20 \mathrm{mM}$ MOPS-KOH, pH 7.5, $80 \mathrm{mM} \mathrm{NaCl}, 10 \mathrm{mM} \mathrm{MgCl}_{2}$, $10 \mathrm{mM} \mathrm{KCl}, 1 \mathrm{mM}$ DTT, $10 \%$ glycerol. Then the corresponding fractions were analyzed by SDS-PAGE and immunoblotting using antisera against Cpn20 or GroES. The positions of the ClpP and co-chaperonin complexes are indicated at the top of the panel. The experiment was repeated three times independently with similar results and a representative result was shown.

\section{Figure 2. Co-chaperonins inhibit ClpP proteolytic activity}

(A) and (C) Degradation of $\beta$-casein was monitored in reactions containing $\beta$-casein (16 $\mu \mathrm{M}), \mathrm{CrClpP}(0.4 \mu \mathrm{M}), \mathrm{EcClpP}(0.4 \mu \mathrm{M}), \mathrm{Cpn} 20(0.4 \mu \mathrm{M}), \mathrm{Cpn} 11 / 20 / 23(0.4 \mu \mathrm{M})$ and ADEP dissolved in DMSO (4 or $18 \mu \mathrm{M})$ ) as indicated. Reactions were performed at $30^{\circ} \mathrm{C}$, aliquots were taken at the indicated time points, and analyzed via SDS-PAGE (15\% gels) and Coomassie staining. Arrowheads indicate the position of $\beta$-casein, stars 
indicate the position of the EcClpP protein and arrows indicate the position of Cpn20. (B) and (D) Densitometric quantification of $\beta$-casein from the reactions shown in (A) and $(\mathbf{C})$, respectively. Shown are mean values from three independent replicates, error bars represent SD. Quantifications were made with Photoshop CS6.

\section{Figure 3. Cryo-EM structure of the Chlamydomonas CIpP complex}

(A) Overview of ClpP-S1 particles. The dimensions are given next to the particles. The cut-away views of the central pore in the two rings of the ClpP core complex are shown.

(B) Overview of ClpP-S2 $\mathrm{C}$ particles. The dimensions are given next to the particles.

(C) Superimposition of EcClpP (PDB ID:1TYF) with density maps of CrClpP-S1. The subunits located in the top ring of EcClpP are shown in light or dark green and the subunits of the bottom ring are shown in yellow or orange. The dotted line indicates the interface between the two rings. Additional densities of $\mathrm{CrClpP}$ not overlapping with EcClp are shown in gray. The most prominent ones are labeled A1, A2 and A3.

\section{Figure 4. Properties of the Chlamydomonas ClpP core complex}

(A) Cartoon presentation of the CrClp core complex. The subunits designated as D1D14 are shown in the left grey cartoon and the corresponding assigned subunits are colored at the right. Subunit ClpP1C occupies positions D8, D10, D13 positions. Subunit ClpP4 occupies positions D3, D5 and D7. Subunit ClpP5 occupies positions D2, D4 and D6. Subunits ClpR1, R2, R3, R4 and R6 occupy single positions at D9, D12, D14, D11 and D1 positions, respectively.

(B) Density map of the ClpP core complex. Densities with assigned Clp subunits are shown in colors, densities that could not be assigned are shown in white.

(C) Subunit composition and arrangement in the top and bottom rings of the ClpP core complex.

(D) Assignment of Clp subunits to the additional density map A1. This region is composed of full-length ClpT4 (cyan), the long C-terminus of ClpR3 (pink), and several amino acids from the C-terminus of ClpR6 (purple). The zoom-in view shows the fitting of these three subunits to the density.

(E) Position of the ClpR4 subunit (turquoise ) in the bottom ring of the ClpP core complex. The long $\mathrm{C}$-terminus of $\mathrm{ClpR} 4$ protrudes to a region next to the top ring. The fitting of ClpR4 is shown in the zoom-in view.

(F) Electrostatic potential of the ClpP core complex. Acidic amino acids are shown in red, basic amino acids in blue.

(G) Hydrophobicity of amino acids at the ClpP core surface. Hydrophobic amino acids are shown in red, hydrophilic amino acids are shown in light blue. The seven hydrophobic binding clefts observed in the top ring of Clp-S1 are encircled by black broken lines.

Figure 5. Cryo-EM structure of the Chlamydomonas ClpP-Cpn11/20/23 complex (A) Sequence alignment of Cpn11, Cpn20-N, Cpn20-C, Cpn23-N, Cpn23-C and GroES. $\mathrm{N}$ and $\mathrm{C}$ indicate $\mathrm{N}$ - and $\mathrm{C}$-terminal GroES-like domains of Cpn20 and Cpn23. The sequence regions of mobile loop and roof are indicated with green boxes. 
721 (B) Superimposition of the Cpn11/20/23 model and the cryo-EM density map. The 722 mobile loops extending from the bottom of the co-chaperonin are shown (left). The 723 enlarged picture shows the roof regions of Cpn23 and Cpn20C.

724 (C) Interaction between $\mathrm{ClpP}$ and co-chaperonin. The central axes of co-chaperonin 725 and $\mathrm{CrClpP}$ are given as black lines. The tilted angle between the axes of co-chaperonin 726 and $\mathrm{CrClpP}$ complex has a value of $4^{\circ}$.

727 (D) Top view oo the extra densities located in the hydrophobic clefts at the top ring of 728 ClpP. The densities colored in red were confidently identified as mobile loops from 729 Cpn11 and Cpn20C. The densities colored in orange might be the mobile loops from 730 Cpn20N. The regions encircled with a broken red line are the two remaining clefts in 731 which no additional densities could be identified.

732 (E) Insertion of the mobile loops of Cpn11 and Cpn20C into the hydrophobic clefts at 733 the surface of the top ring of ClpP.

734 (F) Cartoon presentation of the interaction between the top ring of ClpP and $735 \mathrm{Cpn} 11 / 20 / 23$. Red arrowheads indicate the hydrophobic clefts. High-confidence mobile 736 loops inserted into the clefts are indicated by solid lines, low-confidence mobile loops 737 inserted into the clefts are indicated by broken lines.

738

\section{REFERENCES}

740 1. Cyr, D.M., Hohfeld, J. \& Patterson, C. Protein quality control: U-box-containing E3 ubiquitin ligases join the fold. Trends in biochemical sciences 27, 368-375 (2002).

2. Bukau, B., Weissman, J. \& Horwich, A. Molecular chaperones and protein quality control. Cel/ 125, 443-451 (2006).

744 3. Janska, H., Kwasniak, M. \& Szczepanowska, J. Protein quality control in organellesAAA/FtsH story. Biochimica Et Biophysica Acta (BBA)-Molecular Cell Research 1833, 381-387 (2013).

747 4. Baker, B.M. \& Haynes, C.M. Mitochondrial protein quality control during biogenesis and aging. Trends in biochemical sciences 36, 254-261 (2011).

749 5. Sontag, E.M., Samant, R.S. \& Frydman, J. Mechanisms and Functions of Spatial Protein Quality Control. Annual review of biochemistry 86, 97-122 (2017). 
751 6. Hayer-Hartl, M., Bracher, A. \& Hartl, F.U. The GroEL-GroES Chaperonin Machine: A

752

753

754

755

756

757

758

759

760

761

762

763

764

765

766

767

768

769

770

771

772

Nano-Cage for Protein Folding. Trends in biochemical sciences 41, 62-76 (2016).

7. Kuo, W. et al. CHAPERONIN 20 mediates iron superoxide dismutase (Fe SOD) activity independent of its co-chaperonin role in Arabidopsis chloroplasts. New Phytologist 197, 99-110 (2013).

8. Zhang, X. et al. Arabidopsis co-chaperonin CPN20 antagonizes Mg-chelatase H subunit to derepress ABA-responsive WRKY40 transcription repressor. Science China Life Sciences 57, 11-21 (2014).

9. Zhang, X.-F. et al. Cochaperonin CPN20 negatively regulates abscisic acid signaling in Arabidopsis. Plant molecular biology 83, 205-218 (2013).

10. Rawlings, N.D. et al. The MEROPS database of proteolytic enzymes, their substrates and inhibitors in 2017 and a comparison with peptidases in the PANTHER database. Nucleic Acids Research 46, D624-D632 (2017).

11. Nishimura, K., Kato, Y. \& Sakamoto, W. Chloroplast Proteases: Updates on Proteolysis within and across Suborganellar Compartments. Plant Physio/ 171, 2280-2293 (2016).

12. Gottesman, S. proteases and their targets in escherichia coli. Annual Review of Genetics 30, 465-506 (1996).

13. Wang, J., Hartling, J.A. \& Flanagan, J.M. The structure of ClpP at $2.3 \AA$ resolution suggests a model for ATP-dependent proteolysis. Cell 91, 447-456 (1997).

14. Sauer, R.T. \& Baker, T.A. AAA+ proteases: ATP-fueled machines of protein destruction. Annual review of biochemistry 80, 587-612 (2011).

15. Ripstein, Z.A., Vahidi, S., Houry, W.A., Rubinstein, J.L. \& Kay, L.E. A processive rotary 
mechanism couples substrate unfolding and proteolysis in the ClpXP degradation machinery. Elife 9, e52158 (2020).

16. Lopez, K.E. et al. Conformational plasticity of the ClpAP AAA+ protease couples protein unfolding and proteolysis. Nature Structural \& Molecular Biology, 1-11 (2020).

17. Fei, X. et al. Structures of the ATP-fueled ClpXP proteolytic machine bound to protein substrate. Elife 9 (2020).

18. Gatsogiannis, C., Balogh, D., Merino, F., Sieber, S.A. \& Raunser, S. Cryo-EM structure of the CIpXP protein degradation machinery. Nature structural \& molecular biology $\mathbf{2 6}$, 946-954 (2019).

19. de Sagarra, M.R. et al. Mitochondrial localization and oligomeric structure of HClpP, the human homologue of E. coli ClpP. Journal of molecular biology 292, 819-825 (1999).

20. Kim, J. et al. Structures, Functions, and Interactions of ClpT1 and ClpT2 in the Clp Protease System of Arabidopsis Chloroplasts. Plant Cel/ 27, 1477-1496 (2015).

21. Sjögren, L.L. \& Clarke, A.K. Assembly of the chloroplast ATP-dependent Clp protease in Arabidopsis is regulated by the ClpT accessory proteins. The Plant Cel/23, 322-332 (2011).

22. Olinares, P.D., Kim, J., Davis, J.I. \& van Wijk, K.J. Subunit stoichiometry, evolution, and functional implications of an asymmetric plant plastid ClpP/R protease complex in Arabidopsis. Plant Cel/ 23, 2348-2361 (2011).

23. Stanne, T.M., Pojidaeva, E., Andersson, F.I. \& Clarke, A.K. Distinctive types of ATPdependent Clp proteases in cyanobacteria. The Journal of biological chemistry $\mathbf{2 8 2}$, 
796

797

798

799

800

801

802

803

804

805

806

807

808

809

810

812

24. Majeran, W., Friso, G., van Wijk, K.J. \& Vallon, O. The chloroplast ClpP complex in Chlamydomonas reinhardtii contains an unusual high molecular mass subunit with a large apical domain. The FEBS journa/272, 5558-5571 (2005).

25. Schroda, M. \& Vallon, O. in The Chlamydomonas Sourcebook (Second Edition). (eds. E.H. Harris, D.B. Stern \& G.B. Witman) 671-729 (Academic Press, London; 2009).

26. Derrien, B., Majeran, W., Wollman, F.-A. \& Vallon, O. Multistep processing of an insertion sequence in an essential subunit of the chloroplast ClpP complex. Journal of Biological Chemistry 284, 15408-15415 (2009).

27. Majeran, W., Wostrikoff, K., Wollman, F.A. \& Vallon, O. Role of ClpP in the Biogenesis and Degradation of RuBisCO and ATP Synthase in Chlamydomonas reinhardtii. Plants (Basel) 8 (2019).

28. Ramundo, S. et al. Conditional Depletion of the Chlamydomonas Chloroplast ClpP Protease Activates Nuclear Genes Involved in Autophagy and Plastid Protein Quality Control. Plant Cel/ 26, 2201-2222 (2014).

29. Sjogren, L.L., Stanne, T.M., Zheng, B., Sutinen, S. \& Clarke, A.K. Structural and functional insights into the chloroplast ATP-dependent Clp protease in Arabidopsis. Plant Cell 18, 2635-2649 (2006).

30. Kim, J. et al. Subunits of the plastid ClpPR protease complex have differential contributions to embryogenesis, plastid biogenesis, and plant development in Arabidopsis. Plant Cel/ 21, 1669-1692 (2009).

31. Vahidi, S. et al. An allosteric switch regulates Mycobacterium tuberculosis ClpP1P2 
protease function as established by cryo-EM and methyl-TROSY NMR. Proceedings of the National Academy of Sciences 117, 5895-5906 (2020).

819 32. Derrien, B. et al. The purification of the Chlamydomonas reinhardtii chloroplast ClpP complex: additional subunits and structural features. Plant molecular biology $80,189-$ 202 (2012).

822

33. LaBreck, C.J., May, S., Viola, M.G., Conti, J. \& Camberg, J.L. The Protein Chaperone

34. Gersch, M. et al. AAA+ chaperones and acyldepsipeptides activate the ClpP protease via conformational control. Nat Commun 6, 6320 (2015).

35. Sass, P. et al. Antibiotic acyldepsipeptides activate ClpP peptidase to degrade the cell 828

36. Kirstein, J. et al. The antibiotic ADEP reprogrammes CIpP, switching it from a regulated

37. Zhao, Q. et al. Hetero-oligomeric CPN60 resembles highly symmetric group-I

38. Tsai, Y.-C.C., Mueller-Cajar, O., Saschenbrecker, S., Hartl, F.U. \& Hayer-Hartl, M. chaperonin structure revealed by Cryo-EM. The Plant journal : for cell and molecular biology $98,798-812$ (2019). 

240 (2004).

841 40. Bracher, A., Whitney, S.M., Hartl, F.U. \& Hayer-Hartl, M. Biogenesis and Metabolic Maintenance of Rubisco. Annu Rev Plant Bio/68, 29-60 (2017).

843 41. Weiss, C., Bonshtien, A., Farchi-Pisanty, O., Vitlin, A. \& Azem, A. Cpn20: siamese twins of the chaperonin world. Plant molecular biology 69, 227 (2009). have a novel $\mathrm{Cpn} 10$ in addition to $\mathrm{Cpn} 20$ as co-chaperonins in Arabidopsis thaliana. Journal of Biological Chemistry 276, 29688-29694 (2001).

43. Rizzolo, K. et al. Functional cooperativity between the trigger factor chaperone and the ClpXP proteolytic complex. Nature communications 12, 1-18 (2021).

44. Kim, J. et al. Modified Clp protease complex in the ClpP3 null mutant and consequences for chloroplast development and function in Arabidopsis. Plant Physiol

45. Olinares, P.D., Kim, J. \& van Wijk, K.J. The Clp protease system; a central component of the chloroplast protease network. Biochim Biophys Acta 1807, 999-1011 (2011).

46. Gallastegui, N. \& Groll, M. The 26 S proteasome: assembly and function of a destructive machine. Trends in biochemical sciences 35, 634-642 (2010).

857 47. Maurizi, M.R., Thompson, M.W., Singh, S.K. \& Kim, S.-H. in Methods in enzymology, Vol. 244 314-331 (Elsevier, 1994).

859 48. Derrien, B. \& Vallon, O. One-step Affinity Purification of the Chloroplast ClpP Complex from the Green Alga Chlamydomonas reinhardtii Using the Strep-tagll Epitope Tag. 
49. Bai, C. et al. Protomer Roles in Chloroplast Chaperonin Assembly and Function. Molecular plant 8, 1478-1492 (2015).

50. Mastronarde, D.N. Automated electron microscope tomography using robust prediction of specimen movements. Journal of Structural Biology 152, 36-51 (2005).

51. Rohou, A. \& Grigorieff, N. CTFFIND4: Fast and accurate defocus estimation from electron micrographs. Journal of structural biology 192, 216-221 (2015).

52. Scheres, S.H. RELION: implementation of a Bayesian approach to cryo-EM structure determination. Journal of structural biology 180, 519-530 (2012).

53. Zheng, S.Q. et al. MotionCor2: anisotropic correction of beam-induced motion for improved cryo-electron microscopy. Nature methods 14, 331-332 (2017).

54. Pettersen, E.F. et al. UCSF Chimera--a visualization system for exploratory research and analysis. Journal of computational chemistry 25, 1605-1612 (2004).

874 55. Raman, S. et al. Structure prediction for CASP8 with all-atom refinement using Rosetta. Proteins 77 Suppl 9, 89-99 (2009).

876 56. Song, Y. et al. High-resolution comparative modeling with RosettaCM. Structure 21, 1735-1742 (2013).

878 57. Adams, P.D. et al. PHENIX: a comprehensive Python-based system for macromolecular structure solution. Acta crystallographica. Section D, Biological

881 58. Frenz, B., Walls, A.C., Egelman, E.H., Veesler, D. \& DiMaio, F. RosettaES: a sampling strategy enabling automated interpretation of difficult cryo-EM maps. Nature methods 
883

884 885 886
$14,797-800$ (2017).

59. Wang, R.Y.-R. et al. De novo protein structure determination from near-atomicresolution cryo-EM maps. Nature methods 12, 335-338 (2015).

60. Emsley, P., Lohkamp, B., Scott, W.G. \& Cowtan, K. Features and development of Coot. Acta crystallographica. Section D, Biological crystallography 66, 486-501 (2010).

61. Williams, C.J. et al. MolProbity: More and better reference data for improved all-atom structure validation. Protein science : a publication of the Protein Society 27, 293-315 (2018).

62. DeLano, W.L. Pymol: An open-source molecular graphics tool. CCP4 Newsletter On Protein Crystallography 40, 82-92 (2002). 